\title{
Densidad, velocidad del ultrasonido y módulo dinámico de madera sólida y laminada de Pinus pseudostrobus
}

\section{Density, ultrasound speed and dynamic modulus of solid and laminated wood of Pinus pseudostrobus}

\author{
Isarael Macedo Alquicira' y Javier Ramón Sotomayor Castellanos ${ }^{*}$
}

1 Universidad Michoacana de San Nicolás de Hidalgo. Morelia, Michoacán, México.

* Autor de correspondencia. madera999@yahoo.com

\section{RESUMEN}

La madera laminada potencialmente puede sustituir a la madera sólida para elaborar un material de ingeniería menos variable y más homogéneo. Existe poca información que compare simultáneamente, y en una misma especie, las velocidades del ultrasonido y los módulos dinámicos en madera sólida versus laminada. El objetivo de la investigación fue verificar experimentalmente, en probetas de pequeñas dimensiones, si las características de la madera laminada de Pinus pseudostrobus son similares a las de la madera sólida. Para ello, se calcularon sus densidades y se realizaron pruebas de ultrasonido en las direcciones radial, tangencial y longitudinal. También, se determinaron las velocidades y los módulos dinámicos. Los resultados indican que la densidad de la madera laminada aumenta en comparación con la de la madera sólida. Las velocidades y los módulos dinámicos disminuyen en las direcciones radial y tangencial; en cambio, aumentan para la dirección longitudinal. Se verificó experimentalmente que las magnitudes de la densidad, la velocidad y el módulo dinámico en madera laminada son similares a las de madera sólida de esta especie. La variabilidad en las características estudiadas disminuye como efecto de reconstituir madera sólida. Para las velocidades y los módulos dinámicos de la madera sólida, sus relaciones de anisotropía muestran proporciones similares a las encontradas en la literatura, pero las magnitudes son particulares a cada especie. La investigación aporta datos de referencia y demuestra que la madera sólida y laminada, de $P$. psendostrobus puede ser caracterizada con un método no destructivo.

PALABRAS CLAVE: anisotropía de la madera, caracterización mecánica, contenido de humedad, pruebas no destructivas, variabilidad de la madera.

\section{ABSTRACT}

Laminated wood can potentially replace solid wood to make a less variable and more homogeneous engineered material. There is little information that compares, simultaneously and in the same species, the ultrasound speeds, and the dynamic modules in solid versus laminated wood. The objective of the investigation was to verify experimentally, in small specimens, if the characteristics of Pinus pseudostrobus laminated wood are like those of solid wood. For this, their densities were calculated, and ultrasound tests were performed in the radial, tangential and longitudinal directions. Also, the speeds and dynamic modules were determined. The results indicate that the density of laminated wood increases, compared to that of solid wood. Speeds and dynamic moduli decrease in radial and tangential directions; instead, they increase for the longitudinal direction. It was experimentally verified that the magnitudes of density, velocity and dynamic modulus in laminated wood are like those of solid wood of this species. The variability in the studied characteristics decreases as an effect of reconstituting solid wood. For the speeds and dynamic modules of solid wood, their anisotropy ratios show proportions similar to those reported in the literature, but the magnitudes are specific to each species. The research provides reference data and shows that the solid and laminated wood of $P$. pseudostrobus can be characterized with a non-destructive method.

KEYWORDS: anisotropy of wood, mechanical characterization, moisture content, non-destructive testing, wood variability. 


\section{INTRODUCCIÓN}

Los diámetros de los árboles de especies maderables son cada vez más pequeños, así que las piezas de madera sólida con dimensiones de empleo para aplicaciones industriales son más escasas. Una posible solución tecnológica ante esta problemática es la elaboración de piezas de madera laminada con dimensiones y características similares a las de madera sólida, que puedan sustituirla como material de ingeniería (Kandler, Lukacevic, Zechmeister, Wolff y Füssl, 2018).

Otro problema técnico con respecto a la madera sólida es la variabilidad en las magnitudes de sus módulos dinámicos entre especies, al interior de una misma especie y aun entre piezas de madera aserradas de un mismo árbol (Brémaud, Gril y Thibaut, 2012; Baar, Tippner y Gryc, 2012). De igual forma, los módulos dinámicos varían según las direcciones de anisotropía radial, tangencial y longitudinal del plano leñoso (Dackermann, Elsener y Crews, 2016; Bachtiar, Sanabria, Mittig y Nimes, 2017). Por lo que, para contribuir a solucionar esta problemática, la tendencia en ciencias y tecnología de la madera es la caracterización mecánica de la madera laminada y su comparación con las propiedades de la madera sólida.

Las características mecánicas de la madera laminada dependen, entre otros factores, de las propiedades de la madera de la especie, del tipo de adhesivo, así como del tiempo y la presión aplicados a los laminados durante su fabricación (Bourreau, Aimene, Beauchêne y Thibaut, 2013; Kwon et al., 2014; Dietsch y Tannert, 2015; Liew y Singan, 2016). Existe evidencia empírica de que los módulos dinámicos de la madera laminada son similares a los de la madera sólida (Ribeiro, De Jesus, Lima, A. M. y Lousada, 2009; Gaff y Gáborik, 2014); este argumento coincide con los resultados derivados de pruebas de flexión estática (Ribeiro et al., 2009; Nadir y Nagarajan, 2014), pero no se puede generalizar a todas las especies.

La literatura acerca de la caracterización mecánica de madera sólida y laminada de especies mexicanas incluye información sobre datos obtenidos con pruebas en flexión estática (Araujo et al., 2005; Sotomayor, Adachi, Iida y
Hayashi, 2020) y con métodos no destructivos (Sotomayor, 2020). Entre estos últimos, el método de ultrasonido que permite medir la velocidad de onda a través de la madera y, ponderándola con su densidad, permite calcular el módulo dinámico (Ho, 2017; Ettelaei, Layeghi, Hosseinabadi y Ebrahimi, 2019; Sotomayor, 2019). Empero, existe poca información de pruebas no destructivas que comparen en una misma especie las velocidades del ultrasonido y los módulos dinámicos, simultáneamente en madera sólida versus laminada, distinguiendo al mismo tiempo las direcciones de anisotropía de la madera.

\section{OBJETIVOS}

El objetivo de la investigación fue verificar experimentalmente si las características mencionadas de madera laminada de esta especie son similares a las de madera sólida. La hipótesis de la investigación fue que el laminado de la madera sólida de $P$. pseudostrobus incrementa la magnitud de su densidad, velocidad de ultrasonido, módulo dinámico y, al mismo tiempo, disminuye la variabilidad de estos parámetros. Esta propuesta está limitada a mediciones derivadas de pruebas de ultrasonido en las direcciones radial, tangencial y longitudinal.

\section{MATERIALES Y MÉTODOS}

\section{Materiales}

Se recolectaron piezas de madera de Pinus pseudostrobus Lindl. var. pseudostrobus, en empresas de transformación de productos forestales de la región de Hidalgo y Uruapan, Michoacán, México. El taxón botánico de la madera se identificó en la Facultad de Ingeniería en Tecnología de la Madera, de la Universidad Michoacana de San Nicolás de Hidalgo, en Morelia, México. A partir del material colectado se prepararon 35 probetas de madera sólida y 35 de madera laminada siguiendo el procedimiento propuesto por Sotomayor et al. (2015); la configuración de las probetas se muestra en la figura 1. Para adherir las cinco láminas, se aplicaron $2,5 \mathrm{~kg} \mathrm{~m}^{-2}$ de pegamento de contacto a base de resina de poliacetato de vinilo, repartidos en las cuatro caras interiores de las probetas, correspondientes al plano 
longitudinal-tangencial. El tiempo de prensado fue de 48 horas en ambiente de laboratorio (temperatura de $20{ }^{\circ} \mathrm{C}$ y humedad relativa del aire de $65 \%$ ). Las dimensiones de las probetas fueron $0,05 \mathrm{~m} \times 0,05 \mathrm{~m} \times 0,1 \mathrm{~m}$, orientadas en las direcciones radial, tangencial y longitudinal del plano leñoso. Las probetas se acondicionaron durante seis meses en una cámara con temperatura de $23{ }^{\circ} \mathrm{C}$ y humedad relativa del aire de $60 \%$ hasta que su peso fue constante.

\section{Diseño experimental}

La unidad experimental consistió en dos grupos homogéneos de 35 probetas (réplicas) de madera ( $P$. psendostrobus) seleccionadas al azar: el primer grupo fue madera sólida y el segundo de madera laminada. El proceso de laminado se consideró el tratamiento. El contenido de humedad $(\mathrm{CH})$ se consideró el factor fijo y controlable. Las variables de entrada fueron los parámetros medidos en la madera sólida y las variables de salida fueron los medidos en la madera laminada. Se realizaron siete experimentos de un solo factor de variación (tratamiento de laminado). El

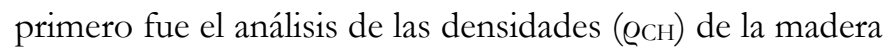
sólida versus laminada. Los otros seis experimentos fueron los análisis de las velocidades ( $\mathrm{v}_{\text {us }}$ y los módulos dinámicos $\left(\mathrm{E}_{\mathrm{us}}\right)$ en tres niveles: direcciones radiales $(\mathrm{R})$, tangenciales (T) y longitudinales (L).

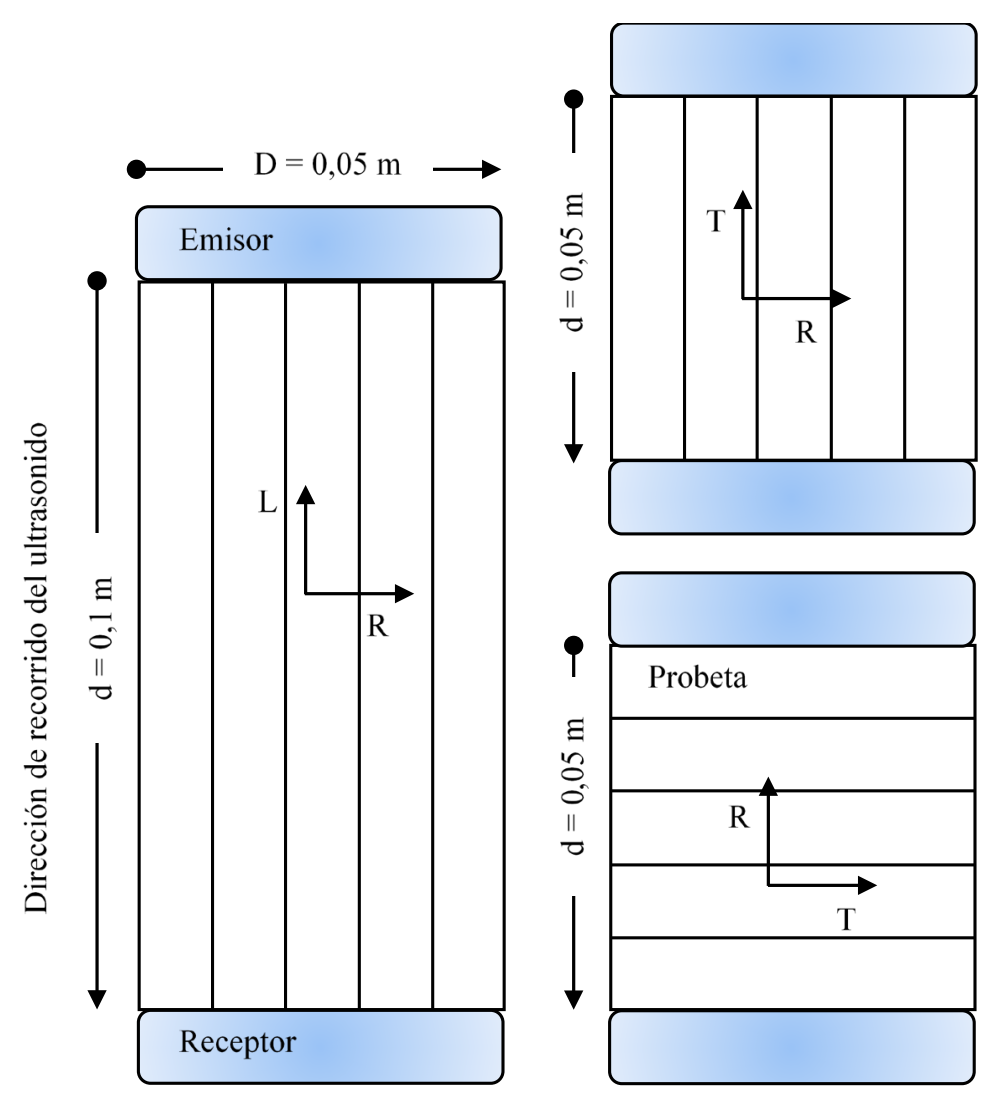

FIGURA 1. Configuración de las pruebas de ultrasonido en madera laminada y maciza de Pinus pseudostrobus. $\mathrm{R}=$ dirección radial; $\mathrm{T}=$ dirección tangencial; $\mathrm{L}$

$=$ dirección longitudinal; $\mathrm{d}=$ distancia de recorrido. 
Tanto para la madera sólida como la laminada, se calcularon las medias $(\mu)$, las desviaciones estándar $(\sigma)$ y los coeficientes de variación $(\mathrm{CV}=\sigma / \mu)$. Asimismo, se realizaron pruebas de normalidad $(\mathrm{SE}=$ sesgo estandarizado; $\mathrm{AE}=$ apuntamiento estandarizado). Cuando estas pruebas indicaron distribuciones anormales, se practicaron análisis de medianas de Kruskal-Wallis (K-W). Cuando las distribuciones resultaron normales, se verificaron las igualdades de varianzas (Ver-var) y se practicaron análisis de varianzas (Anova). Para todas las pruebas el nivel de significancia fue de $95 \%(\alpha=0,05)$. Por lo tanto, el criterio de demarcación fue aceptar una diferencia estadísticamente significativa para valores $\mathrm{P}_{(\alpha}=$ $0,05) \leq 0,05$. Se verificó la hipótesis nula que propone que el laminado no influye en el parámetro calculado mediante $\mathrm{H}_{0}: \mu_{1}=\mu_{2}$ donde $\mathrm{H}_{0}=$ hipótesis nula; $\mu_{1}=$ media de la madera sólida (densidad, velocidad, módulo dinámico); $\mu_{2}$ $=$ media de la madera laminada (densidad, velocidad, módulo dinámico). Para realizar los cálculos estadísticos se utilizó el paquete estadístico Statgraphicsß.

\section{Pruebas de laboratorio}

La densidad de la madera se determinó por la razón entre el peso y el volumen de la probeta al momento del ensayo de acuerdo con la norma ISO 13061-2:2014 (International Organization for Standardization [ISO], 2014b). El contenido de humedad de la madera se calculó por el método de diferencia de pesos húmedo y seco, según la norma ISO 13061-1:2014 (ISO, 2014a), en 20 probetas adicionales: 10 de madera sólida y 10 de laminada. Sus dimensiones fueron $0,05 \mathrm{~m} \times 0,05 \mathrm{~m} \times 0,02 \mathrm{~m}(\mathrm{en} \mathrm{R}, \mathrm{T}, \mathrm{L})$. El contenido de humedad de la madera sólida fue 11,7\% $(\mathrm{CV}=2 \%)$ y el de la madera laminada $10,4 \%(\mathrm{CV}=3 \%)$. Todas las probetas se elaboraron con madera sana y se revisó que estuviesen libres de anomalías de crecimiento como nudos, rajaduras y desviación de la fibra.

Las pruebas de ultrasonido consistieron en medir el tiempo de transmisión del impulso en las direcciones radial, tangencial y longitudinal con el aparato V-Meter MK IV (Frecuencia $=54 \mathrm{kHz}$ ) marca James Instruments ${ }^{\circledR}$ (Fig. 2).
Las velocidades del ultrasonido ( $\mathrm{v}_{\text {us }}$ ) se calcularon con las distancias de recorrido (d) divididas entre los intervalos de los tiempos de transmisión (Fig. 1). Los módulos dinámicos ( $\mathrm{E}_{\mathrm{us}}$ ) se calcularon con la fórmula (1) (Ettelaei et al., 2019):

$$
\mathrm{E}_{\mathrm{us}}=\varrho_{\mathrm{CH}} \mathrm{v}_{\mathrm{us}}^{2}
$$

donde:

$\mathrm{E}_{\mathrm{us}}=$ módulo dinámico $\left(\mathrm{N} \mathrm{m}^{-2}\right)$

$\varrho_{\mathrm{CH}}=$ densidad $\left(\mathrm{kg} \mathrm{m}^{-3}\right)$

$\mathrm{v}_{\mathrm{us}}=$ velocidad del ultrasonido $\left(\mathrm{m} \mathrm{s}^{-1}\right)$

\section{RESULTADOS Y DISCUSIÓN}

Densidad de la madera

Los resultados de las pruebas de ultrasonido, resumidos en la tabla 1 , muestran que la densidad de la madera laminada aumenta $7 \%$ en comparación con la de la madera sólida. Este resultado indica, experimentalmente, que el proceso de laminado incrementa las magnitudes de las densidades. No obstante, el análisis de varianza de la madera sólida versus laminada no reveló diferencias estadísticamente significativas (Tabla 2). Como efecto del tratamiento de laminado, la variabilidad de la densidad de la madera laminada disminuye $67 \%$ respecto a la madera sólida. Así, este resultado sugiere que el laminado de la madera de $P$. psendostrobus disminuye la variabilidad de las densidades. Estos corolarios son similares a los señalados por Sotomayor (2018) quien observó incrementos en la densidad de la madera laminada respecto a la madera sólida de $10 \%$ para Enterolobium cyclocarpum, de $1 \%$ para Tabebuia rosea y para Juglans pyriformis de 6\%. En el mismo contexto, informa una disminución en la variabilidad de $25 \%, 35 \%$ y $16 \%$ respectivamente.

Este aumento aparente en la densidad de la madera laminada puede ser explicado tanto por la variabilidad natural al interior de la misma especie, como por la contribución del peso del adhesivo incorporado en la madera laminada. Por su parte, la disminución en la variabilidad de la densidad después del proceso de laminado parece ser ocasionada por el reacomodo y la combinación de las piezas que conforman la madera laminada (Bourreau et al., 2013). 

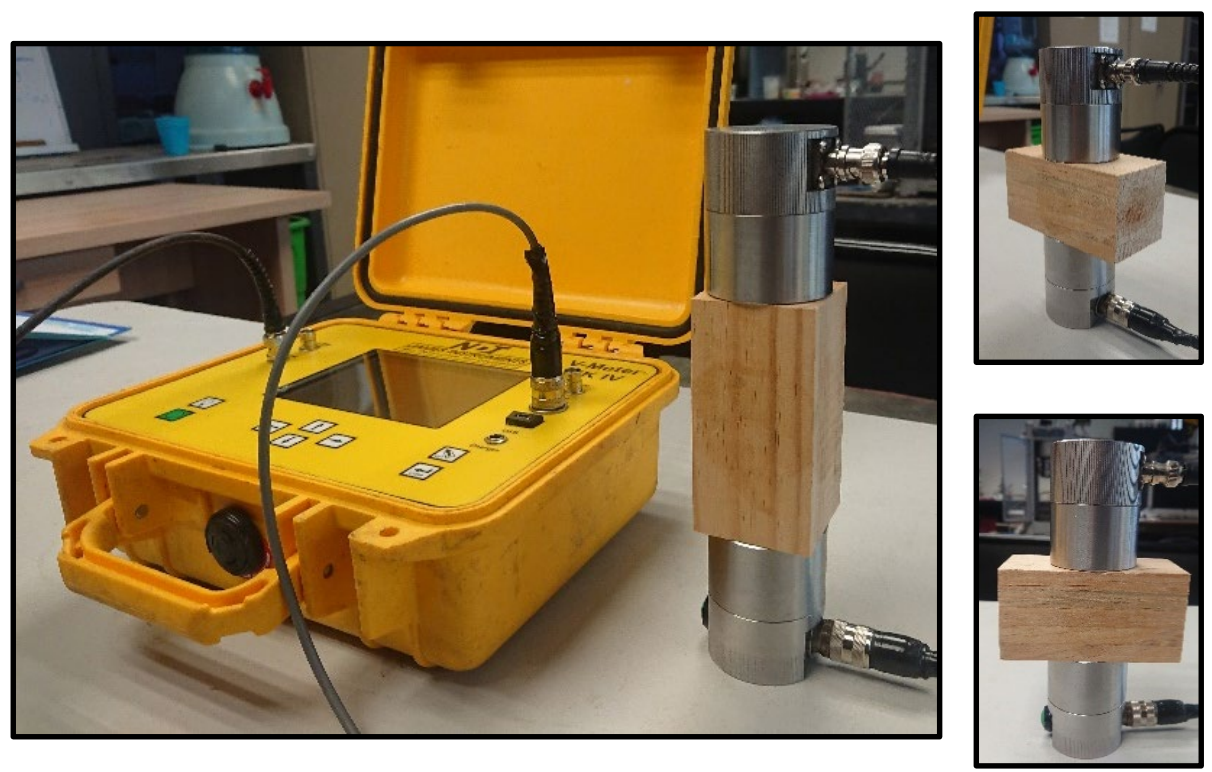

FIGURA 2. Pruebas de ultrasonido en madera laminada y maciza de Pinus psendostrobus.

TABLA 1. Resultados de pruebas de ultrasonido en madera de Pinus pseudostrobus.

\section{Madera sólida}

\begin{tabular}{|c|c|c|c|c|c|c|c|}
\hline & $\begin{array}{c}\rho_{C H} \\
\left(k g m^{-3}\right)\end{array}$ & $\begin{array}{l}V_{\text {us }} R \\
\left(m s^{-1}\right)\end{array}$ & $\begin{array}{c}V_{u s} T \\
\left(m s^{-1}\right)\end{array}$ & $\begin{array}{l}V_{\text {us }} L \\
\left(m s^{-1}\right)\end{array}$ & $\begin{array}{c}E_{\text {us }} R \\
\left(M N m^{-2}\right)\end{array}$ & $\begin{array}{c}E_{u s} T \\
\left(M N m^{-2}\right)\end{array}$ & $\begin{array}{c}E_{u s} L \\
\left(M N m^{-2}\right)\end{array}$ \\
\hline$\mu$ & 479 & 2300 & 1652 & 4270 & 2569 & 1331 & 8947 \\
\hline$\sigma$ & 34 & 233 & 212 & 511 & 536 & 412 & 2435 \\
\hline $\mathrm{CV}$ & 7 & 10 & 13 & 12 & 21 & 31 & 27 \\
\hline \multicolumn{8}{|c|}{ Madera laminada } \\
\hline & $\rho_{\mathrm{CH}}$ & Vus $R$ & Vus $T$ & Vus $L$ & Eus $R$ & Eus $T$ & Eus $L$ \\
\hline & $\left(\mathrm{kg} \mathrm{m}^{-3}\right)$ & $\left(\mathrm{m} \mathrm{s}^{-1}\right)$ & $\left(\mathrm{m} \mathrm{s}^{-1}\right)$ & $\left(\mathrm{m} \mathrm{s}^{-1}\right)$ & $\left(\mathrm{MN} \mathrm{m}^{-2}\right)$ & $\left(\mathrm{MN} \mathrm{m}^{-2}\right)$ & $\left(\mathrm{MN} \mathrm{m}^{-2}\right)$ \\
\hline$\mu$ & 514 & 2199 & 1295 & 5919 & 2493 & 870 & 17996 \\
\hline$\sigma$ & 12 & 132 & 134 & 112 & 308 & 169 & 764 \\
\hline $\mathrm{CV}$ & 2 & 6 & 10 & 2 & 12 & 19 & 4 \\
\hline
\end{tabular}

Diferencias entre medias laminada/sólida (\%)

\begin{tabular}{|c|c|c|c|c|c|c|c|}
\hline - & $\rho_{\mathrm{CH}}$ & Vus $R$ & Vus $T$ & Vus L & Eus $R$ & Eus $T$ & Eus $L$ \\
\hline- & +7 & -4 & -22 & +39 & -3 & -35 & +101 \\
\hline \multicolumn{8}{|c|}{ Diferencias entre coeficientes de variación laminada/sólida (\%) } \\
\hline 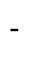 & $\rho \mathrm{CH}$ & Vus $R$ & Vus $T$ & Vus $L$ & Eus $R$ & Eus $T$ & Eus $L$ \\
\hline- & -67 & -41 & -19 & -84 & -41 & -37 & -84 \\
\hline
\end{tabular}

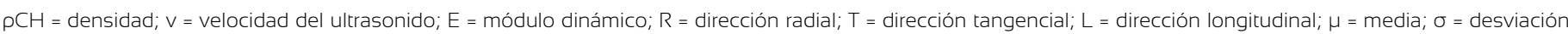
estándar; $C V$ = coeficiente de variación en porciento 
TABLA 2. Resultados de las pruebas de normalidad, verificación y análisis de varianza y Kruskal-Wallis de la densidad de la madera sólida versus laminada obtenida mediante pruebas de ultrasonido.

\begin{tabular}{|c|c|c|c|c|c|}
\hline & $\begin{array}{c}S E \\
-\end{array}$ & $\begin{array}{c}A E \\
-\end{array}$ & $\begin{array}{l}\text { Ver-Var } \\
P_{(\alpha=0,05)}\end{array}$ & $\begin{array}{c}\text { Anova } \\
P_{(\alpha=0,05)}\end{array}$ & $\begin{array}{c}K-W \\
P_{(\alpha=0,05)}\end{array}$ \\
\hline$\rho_{\mathrm{CH}}$ Madera sólida & $-1,786$ & 0,094 & $<0,001$ & $<0,001^{*}$ & - \\
\hline$\rho \subset \mathrm{cH}$ Madera laminada & 0,005 & $-0,721$ & - & - & - \\
\hline Vus R Madera sólida & $-4,146$ & 4,194 & - & - & $<0,001^{*}$ \\
\hline Vus R Madera laminada & $-2,042$ & 0,925 & -- & - & \\
\hline Vus T Madera sólida & 5,359 & 5,700 & - & - & $<0,001^{*}$ \\
\hline Vus T Madera laminada & $-2,659$ & 1,942 & - & - & - \\
\hline Vus L Madera sólida & $-1,078$ & $-0,968$ & $<0,001^{*}$ & $<0,001^{*}$ & - \\
\hline Vus L Madera laminada & 0,176 & $-1,036$ & - & - & - \\
\hline Eus R Madera sólida & $-3,158$ & 1,502 & - & - & $0.073^{* *}$ \\
\hline Eus R Madera laminada & $-1,237$ & 0,305 & - & - & - \\
\hline Eus T Madera sólida & 5,954 & 8,523 & - & - & $<0,001^{*}$ \\
\hline Eus T Madera laminada & $-1,801$ & 0,961 & - & - & - \\
\hline Eus L Madera sólida & $-0,762$ & $-1,059$ & $<0,001^{*}$ & $<0,001^{*}$ & - \\
\hline Eus L Madera laminada & 0,246 & $-0,335$ & - & - & - \\
\hline
\end{tabular}

$\mathrm{PCH}$ = densidad; $\mathrm{V}=$ velocidad del ultrasonido; $\mathrm{E}=$ módulo dinámico; $\mathrm{R}=$ dirección radial; $\mathrm{T}$ = dirección tangencial; $\mathrm{L}$ = dirección longitudinal; $\mathrm{SE}$ = sesgo estandarizado; $\mathrm{AE}$ = apuntamiento estandarizado; K-W = Kruskal-Wallis; Ver-var = verificación de varianza; Anova = análisis de varianza; * diferencia significativa para 95\% de confiabilidad $* *$ no existe diferencia significativa para $95 \%$ de confiabilidad.

\section{Velocidad de trasmisión del ultrasonido}

Se encontró que las velocidades de trasmisión del ultrasonido disminuyen en las direcciones radial (4\%) y tangencial (22\%). En cambio, aumentan para la dirección longitudinal (39\%). De aquí que la hipótesis referente al incremento en las velocidades como resultado del laminado se válida para la dirección longitudinal, pero discrepa para las direcciones radial y tangencial. Esta diferencia puede ser ocasionada por la presencia en la madera laminada de cuatro capas de adhesivo que funcionan como revestimientos aislantes y puede alterar la transmisión del ultrasonido (Dietsch y Tannert, 2015). Los coeficientes de variación de las velocidades disminuyen en la madera laminada en las tres direcciones de anisotropía un promedio de 48\% (Tabla 1). Este resultado verifica la hipótesis sobre la disminución de la variabilidad de las velocidades de la madera de P. pseudostrobus por el efecto del laminado.

Las pruebas estadísticas indican que las trasmisiones del ultrasonido no corresponden a distribuciones normales para las direcciones radial y tangencial (Tabla 2). Así que, para ambas direcciones, las pruebas de diferencias de medianas resultan en diferencias significativas $\mathrm{p}<0.05$ ) entre las velocidades de la madera sólida y las de la madera laminada.

En la dirección radial de la madera sólida, el ultrasonido se transmite a través de las diferentes capas de crecimiento de la madera. De manera análoga, en la madera laminada viaja a través de las diferentes láminas que forman la sección transversal de las probetas. En cambio, en la dirección tangencial, el ultrasonido viaja de forma paralela a las capas de crecimiento de la madera sólida y, en el caso 
de la madera laminada, viaja en la dirección tangencial de las láminas y los planos de pegamento entre ellas. Debido a esta estructura natural en la madera sólida, y/o artificial en la laminada, cualquier pequeña anomalía en los tejidos leñosos o en el acomodo de las láminas puede alterar el tiempo de recorrido del ultrasonido. Aunado a esto, hay que considerar la variabilidad y heterogeneidad en la estructura anatómica de la madera en probetas con dimensiones de cinco centímetros en las aristas de su sección transversal, valores mínimos para piezas de madera para uso estructural.

Para la dirección longitudinal, el análisis de varianza también indica diferencias significativas entre las velocidades de la madera sólida y las de laminada. El ultrasonido viaja en paralelo a la estructura tubular de las células alineadas en la dirección longitudinal de la madera sólida. En el mismo sentido, el arreglo artificial de la madera laminada hace más homogénea la estructura material en esta dirección. Para ilustrar este efecto, en la figura 3 se observa que la dispersión de las densidades y las velocidades de la madera sólida son más amplias en comparación con la dispersión de la madera laminada. La correlación de la velocidad, en función de la densidad para la madera sólida, muestra una amplia dispersión con una tendencia positiva y un coeficiente de determinación débil $\left(\mathrm{R}^{2}=0,45\right)$. En cambio, en la correlación correspondiente a la madera laminada, la tendencia es prácticamente nula $\left(\mathrm{R}^{2}=0,01\right)$. Sin embargo, se observa un agrupamiento en los valores, lo que verifica la disminución de su variabilidad.

Para ubicar las magnitudes de los resultados de esta investigación respecto a datos de la bibliografía, la tabla 3 detalla las densidades, velocidades y módulos dinámicos de 18 especies del género Pinus obtenidos por Sotomayor, Guridi y García (2010b). Las magnitudes de las velocidades radial, tangencial y longitudinal, de madera sólida y laminada de esta investigación, se sitúan al interior de los diferentes intervalos de valores de referencia. En el mismo sentido, Dackermann et al. (2016) observaron para Corymbia maculata (ecH $=1060 \mathrm{~kg} \mathrm{~m}^{-3}$ ) velocidades del ultrasonido de $\mathrm{v}_{\mathrm{us}} \mathrm{R}=1982 \mathrm{~m} \mathrm{~s}^{-1}, \mathrm{v}_{\text {us }} \mathrm{T}=1760 \mathrm{~m} \mathrm{~s}^{-1} \mathrm{y} \mathrm{v}_{\text {us }} \mathrm{L}=5555 \mathrm{~m} \mathrm{~s}^{-1}$; y para Eucalyptus microcorys ( $\varrho \mathrm{cH}=1090 \mathrm{~kg} \mathrm{~m}^{-3}$ ) de $\mathrm{v}_{\text {us }} \mathrm{R}=$ $2095 \mathrm{~m} \mathrm{~s}^{-1}, \mathrm{v}_{\text {us }} \mathrm{T}=1820 \mathrm{~m} \mathrm{~s}^{-1} \mathrm{y} \mathrm{v}_{\text {us }} \mathrm{L}=5137 \mathrm{~m} \mathrm{~s}^{-1}$; valores cercanos a los de esta investigación y a los presentados en la tabla 3. Las relaciones de anisotropía de las velocidades para la madera sólida son $\mathrm{v}_{\text {us }} \mathrm{T} / \mathrm{v}_{\text {us }} \mathrm{R}=0,72 \mathrm{y} \mathrm{v}$ us $\mathrm{L} / \mathrm{v}_{\mathrm{us}} \mathrm{R}$ $=1,86$; mientras que para la madera laminada son $\mathrm{v}_{\mathrm{us}} \mathrm{T} / \mathrm{v}_{\mathrm{us}}$ $\mathrm{R}=0,59 \mathrm{y} \mathrm{v}_{\text {us }} \mathrm{L} / \mathrm{v}_{\text {us }} \mathrm{R}=2,69$.

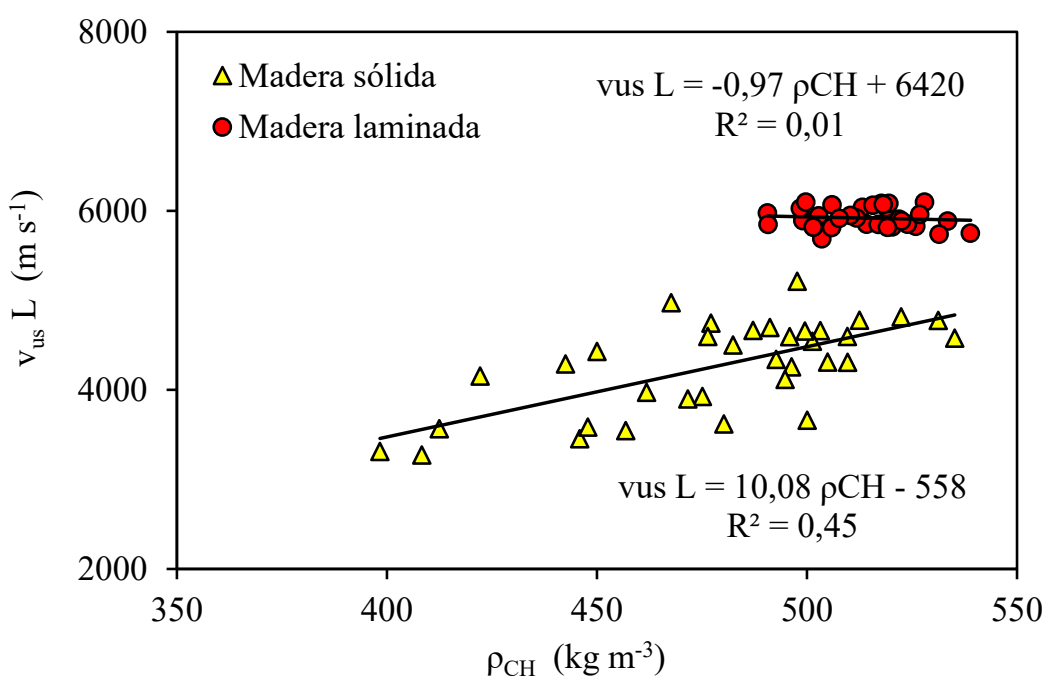

FIGURA 3. Correlaciones entre velocidad longitudinal de ultrasonido de madera de Pinus pseudostrobus y su densidad. 
TABLA 3. Densidad, velocidad del ultrasonido y módulo dinámico de la madera de diferentes especies (Datos de la bibliografía, Sotomayor et al., 2010b).

\begin{tabular}{|c|c|c|c|c|c|c|c|}
\hline $\begin{array}{c}\text { Género Pinus } \\
\text { Especies }\end{array}$ & $\begin{array}{c}\rho_{C H} \\
\left(\mathrm{~kg} \mathrm{~m}^{-3}\right)\end{array}$ & $\begin{array}{l}v_{\text {us }} R \\
\left(m s^{-1}\right)\end{array}$ & $\begin{array}{c}v_{u s} T \\
\left(m s^{-1}\right)\end{array}$ & $\begin{array}{l}V_{\text {us }} L \\
\left(m s^{-1}\right)\end{array}$ & $\begin{array}{c}E_{\text {us }} R \\
\left(M N m^{-2}\right)\end{array}$ & $\begin{array}{c}E_{u s} T \\
\left(M N m^{-2}\right)\end{array}$ & $\begin{array}{c}E_{\text {us }} L \\
\left(M N m^{-2}\right)\end{array}$ \\
\hline P. arizonica & 461 & 3713 & 1594 & 6020 & 7107 & 1309 & 18682 \\
\hline P. ayacahuite & 398 & 2206 & 1370 & 5986 & 2169 & 836 & 15974 \\
\hline P. cembroides & 525 & 3690 & 1108 & 3321 & 7994 & 721 & 6475 \\
\hline P. coulteri & 419 & 2108 & 984 & 6023 & 2082 & 453 & 16997 \\
\hline P. douglasiana & 405 & 1845 & 1283 & 5993 & 1543 & 747 & 16280 \\
\hline P. durangensis & 390 & 3423 & 1581 & 6547 & 5112 & 1091 & 18695 \\
\hline P. lawsonii & 586 & 2798 & 1751 & 4503 & 5127 & 2009 & 13283 \\
\hline P. martinezii & 539 & 1418 & 1259 & 6222 & 1213 & 957 & 23353 \\
\hline P. maximinoi & 432 & 2328 & 1581 & 6145 & 2618 & 1207 & 18238 \\
\hline P. michoacana & 463 & 3663 & 1080 & 6518 & 6949 & 604 & 21999 \\
\hline P. montezumae & 497 & 1883 & 1851 & 6307 & 1974 & 1906 & 22133 \\
\hline P. oocarpa & 548 & 2200 & 1360 & 5338 & 2966 & 1134 & 17462 \\
\hline P. patula & 496 & 3673 & 1204 & 7187 & 7482 & 804 & 28637 \\
\hline P. ponderosa & 490 & 3563 & 1583 & 5532 & 6964 & 1374 & 16787 \\
\hline P. pringlei & 580 & 2579 & 1868 & 6390 & 4315 & 2265 & 26495 \\
\hline P.pseudostrobus & 436 & 3403 & 959 & 6514 & 5654 & 449 & 20711 \\
\hline P. quadrifolia & 678 & 2260 & 1369 & 3857 & 3872 & 1421 & 11281 \\
\hline P. teocote & 638 & 2881 & 1636 & 6075 & 5925 & 1910 & 26334 \\
\hline
\end{tabular}

pCH = Densidad; $v$ = Velocidad del ultrasonido; E = Módulo dinámico; R = Dirección radial; $T$ = Dirección tangencial; L = Dirección longitudinal.

Estos resultados son similares a los que Sotomayor, García, Moya Lara y Olguín Cerón (2010a), determinaron con ultrasonido para P. michoacana: $\mathrm{v}_{\text {us }} \mathrm{T} / \mathrm{v}_{\text {us }} \mathrm{R}=0,64 \mathrm{y} \mathrm{v}$ us $\mathrm{L} / \mathrm{v}_{\mathrm{us}}$ $\mathrm{R}=2,74$; para $P$. douglasiana: $\mathrm{v}_{\mathrm{us}} \mathrm{T} / \mathrm{v}_{\mathrm{us}} \mathrm{R}=0,65 \mathrm{y} \mathrm{v}_{\mathrm{us}} \mathrm{L} / \mathrm{v}_{\mathrm{us}}$ $\mathrm{R}=3,04 ; \mathrm{y}$ para $P$. pringlei: $\mathrm{v}_{\text {us }} \mathrm{T} / \mathrm{v}_{\text {us }} \mathrm{R}=0,78 \mathrm{y} \mathrm{v}_{\text {us }} \mathrm{L} / \mathrm{v}_{\text {us }} \mathrm{R}$ $=3,01$. Si bien las relaciones muestran proporciones similares, las magnitudes son particulares a cada especie. Los valores precedentes son próximos a los calculados a partir de las velocidades por Dackermann et al. (2016) y detallados anteriormente para Corymbia maculata y Eucalyptus microcorys.

\section{Módulos dinámicos}

Los módulos dinámicos de la madera laminada disminuyen en la dirección radial (3\%) y en la tangencial (35\%) (Tabla 1). En contraste, los módulos dinámicos aumentan en la dirección longitudinal (101\%). De manera análoga a las velocidades, los coeficientes de variabilidad de los módulos dinámicos disminuyen en las tres direcciones.

Caso particular son los módulos dinámicos en la dirección radial cuya diferencia aritmética entre los coeficientes de variabilidad es de 3\%. Esto se refleja en el resultado de la prueba de medianas, la cual indica que no existe una diferencia estadísticamente significativa entre los módulos dinámicos de la madera sólida y la laminada (Tabla 2). Es decir, el proceso de laminado no modifica de manera significativa el módulo dinámico en esta dirección, pero sí disminuye su variación (Tabla 1). De manera diferente, para las direcciones tangencial y longitudinal las pruebas de hipótesis indican diferencias significativas. Es notorio que las distribuciones de las muestras de los módulos dinámicos radiales y tangenciales de la madera sólida son anormales, al igual que las distribuciones de las velocidades correspondientes. No obstante, se observan diferencias estadísticamente significativas para estas dos direcciones. 
Una interpretación gráfica de estos resultados se ejemplifica en la figura 4 donde se presentan las correlaciones entre módulos dinámicos longitudinales y densidades de la madera sólida y laminada. De manera análoga al análisis de las velocidades (Fig. 3), el intervalo de las magnitudes de los módulos dinámicos de la madera laminada disminuye de manera importante, resultado que coincide con la diferencia de 101\% entre medias de laminada versus sólida, registrada en la tabla 1. En este sentido, ambas correlaciones tienen tendencias similares pero el coeficiente de determinación correspondiente a la madera laminada es bajo en comparación con el de la madera sólida que es medio. Para el caso específico de los módulos longitudinales, estos resultados sugieren que el laminado de la madera sólida de P. pseudostrobus aumenta y homogeniza sus magnitudes.

La disminución en los módulos dinámicos en las direcciones radial y tangencial de la madera laminada es similar a la referida por Nadir y Nagarajan (2014) para módulos de elasticidad determinados en flexión estática en probetas de pequeñas dimensiones de madera sólida de Hevea brasiliensis ( $\varrho \mathrm{CH}=605 \mathrm{~kg} \mathrm{~m}^{-3}$ ). En efecto, estos autores mencionan una disminución de 5\% en madera laminada en comparación con la madera sólida, pero no encontraron diferencias estadísticamente significativas entre el módulo de elasticidad estático de la madera sólida y el de laminada. En contraste, para probetas de pequeñas dimensiones de
Pinus pinaster ( $\left.\varrho_{\mathrm{CH}}=550 \mathrm{~kg} \mathrm{~m}^{-3}\right)$, Ribeiro et al. (2009) informan un incremento de $2 \%$ en los módulos de elasticidad en flexión estática de la madera laminada.

Por otra parte, estudiando probetas de pequeñas dimensiones de madera laminada compuesta de Abies religiosa $\left(\varrho \mathrm{CH}=415 \mathrm{~kg} \mathrm{~m}^{-3}\right)$, Fraxinus ubdei $(\varrho \mathrm{CH}=623 \mathrm{~kg}$ $\left.\mathrm{m}^{-3}\right)$ y Alnus acuminata $\left(\varrho_{\mathrm{CH}}=565 \mathrm{~kg} \mathrm{~m}^{-3}\right)$, Sotomayor y Ruiz (2017) concluyen que el módulo dinámico de la madera laminada, determinado con ultrasonido en la dirección longitudinal $\left(\mathrm{E}_{\mathrm{us}}=12706 \mathrm{MN} \mathrm{m}^{-2}\right)$, mejora en comparación con el de madera sólida de $A$. religiosa $\left(\mathrm{E}_{\mathrm{us}}=7258 \mathrm{MN} \mathrm{m}^{-}\right.$ ${ }^{2}$ ), es menor al de F. ubdei $\left(\mathrm{E}_{\mathrm{us}}=13183 \mathrm{MN} \mathrm{m}^{-2}\right)$ y similar al de $A$. acuminata $\left(\mathrm{E}_{\mathrm{us}}=12833 \mathrm{MN} \mathrm{m}^{-2}\right)$. Estos investigadores sugieren considerar los resultados caso por caso. El incremento o decremento del módulo dinámico depende, por una parte, de la configuración de las pruebas y, por otra, de factores de variación como el adhesivo aplicado, la especie y/o la combinación de diferentes especies utilizadas, así como el acomodo y las características de los componentes de la madera laminada. En el mismo sentido, Burdurlu, Kilic, Ilce y Ozan (2007) obtuvieron resultados combinados entre el módulo de elasticidad estático de Fagus orientalis ( $\left.\varrho_{\mathrm{CH}}=703 \mathrm{~kg} \mathrm{~m}^{-3}\right)$ y Populus nigra $\left(\varrho_{\mathrm{CH}}=400 \mathrm{~kg}\right.$ $\mathrm{m}^{-3}$ ) y los módulos correspondientes a diferentes combinaciones de estas especies en probetas de pequeñas dimensiones de madera laminada.

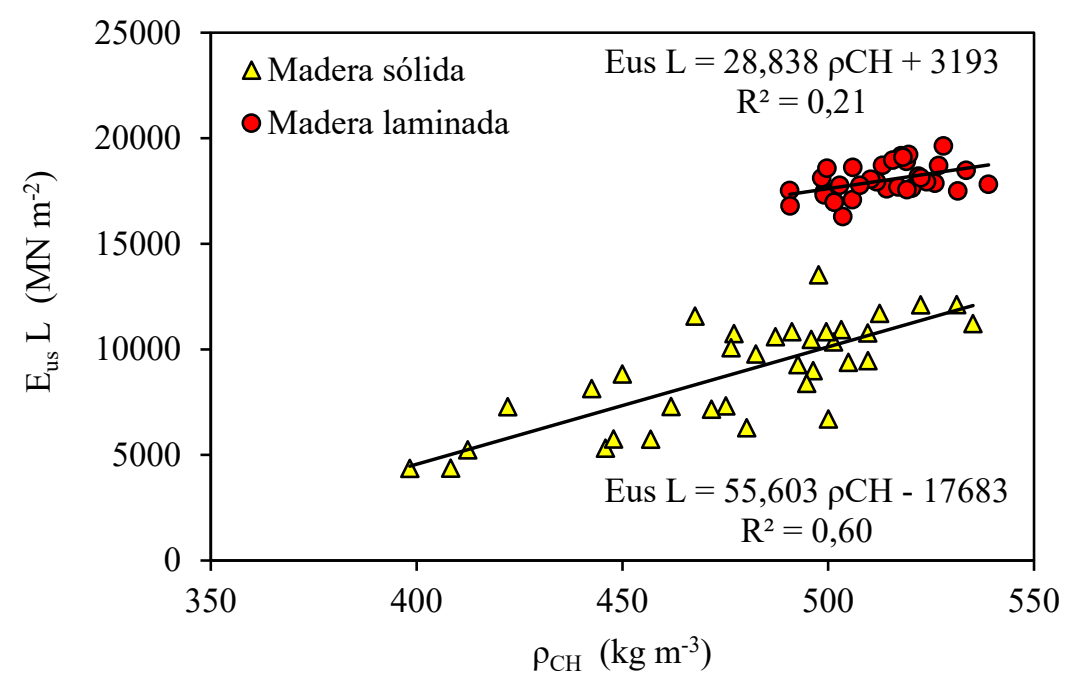

FigurA 4. Correlaciones entre módulo dinámico longitudinal de madera de Pinus pseudostrobus y su densidad. 
Las relaciones de anisotropía de la madera solida de $P$. pseudostrobus (Tabla 1) son $\mathrm{E}_{\mathrm{us}} \mathrm{T} / \mathrm{E}_{\mathrm{us}} \mathrm{R}=0,52$ y $\mathrm{E}_{\mathrm{us}} \mathrm{L} / \mathrm{E}_{\mathrm{us}}$ $\mathrm{R}=3,50$. De manera análoga a los resultados de las velocidades publicados por Sotomayor et al. (2010a), los resultados para los módulos dinámicos para especies del género Pinus muestran relaciones de anisotropía similares en las direcciones tangencia/radial, pero menores para las direcciones longitudinal/tangencial, para P. michoacana: $\mathrm{E}_{\mathrm{us}}$ $\mathrm{T} / \mathrm{E}_{\mathrm{us}} \mathrm{R}=0,40$ y $\mathrm{E}_{\mathrm{us}} \mathrm{L} / \mathrm{E}_{\mathrm{us}} \mathrm{R}=7,75$; para P. douglasiana: $\mathrm{E}_{\mathrm{us}}$ $\mathrm{T} / \mathrm{E}_{\mathrm{us}} \mathrm{R}=0,46$ y $\mathrm{E}_{\text {us }} \mathrm{L} / \mathrm{E}_{\mathrm{us}} \mathrm{R}=9,25$; y para P. pringlei: $\mathrm{E}_{\mathrm{us}}$ $\mathrm{T} / \mathrm{E}_{\text {us }} \mathrm{R}=0,56$ y $\mathrm{E}_{\text {us }} \mathrm{L} / \mathrm{E}_{\mathrm{us}} \mathrm{R}=7,20$. Por su parte, para el caso de madera clasificada para uso estructural, Ho (2017) registra anisotropías de los módulos dinámicos por ultrasonido en madera sólida de Larix kaempferi ( $\varrho_{\mathrm{CH}}=578$ $\mathrm{kg} \mathrm{m}^{-3}$ ) de $\mathrm{E}_{\text {us }} \mathrm{T} / \mathrm{E}_{\text {us }} \mathrm{R}=0,72$ y $\mathrm{E}_{\text {us }} \mathrm{L} / \mathrm{E}_{\text {us }} \mathrm{R}=6,11$. En el mismo contexto, para madera laminada, las relaciones de anisotropía de esta investigación son $\mathrm{E}_{\mathrm{us}} \mathrm{T} / \mathrm{E}_{\mathrm{us}} \mathrm{R}=0,35 \mathrm{y}$ $\mathrm{E}_{\mathrm{us}} \mathrm{L} / \mathrm{E}_{\mathrm{us}} \mathrm{R}=7,22$. Los resultados son similares a los mencinados para madera sólida por los autores citados anteriormente.

A manera de síntesis, se puede inferir que las relaciones de anisotropía para madera sólida, publicadas en las investigaciones referidas, muestran proporciones similares a las de este trabajo, pero las magnitudes son particulares a cada especie. Empero, en la literatura revisada no se encontraron artículos que informen los datos de las relaciones de anisotropía para la madera laminada. Para posicionar los resultados de esta investigación respecto a los observados para madera sólida de maderas mexicanas del género Pinus por Sotomayor et al. (2010b), detallados en la tabla 3 , se presentan en la figura 5 las dispersiones de los módulos dinámicos en función de las densidades para las dirección radial, tangencial y longitudinal. En las tres direcciones, las magnitudes de esta investigación para la madera sólida y laminada se posicionan al interior de la nube de datos correspondiente a los resultados referenciados.

No obstante que los valores de los módulos dinámicos se sobreponen en la misma nube de datos, se observan diferencias entre especies y tipos de madera. Esta diversidad puede explicarse por la variabilidad natural en la magnitud de las características físicas que existe entre especies y al interior de una especie (Perré, Almeida, Ayouz y Frank 2016), por la heterogeneidad anatómica (Schubert, Gsell, Dual, Motavalli y Niemz, 2009), por la anisotropía material (Brémaud et al. 2011) y por la higroscopicidad del tejido leñoso (Mvondo, Meukam, Jeong, Meneses y Nkeng 2017). La diversidad en las propiedades físico-mecánicas de la madera también depende tanto de factores genéticos como ambientales y se encuentra en todos los niveles de gimnospermas y angiospermas, entre árboles de climas tropicales y templados, entre poblaciones de una especie determinada, entre árboles de una población y, finalmente, en ubicaciones de muestreo dentro de un solo árbol (Zhang et al. 2011).

Una causa adicional que provoca la variabilidad del módulo dinámico de la madera son las diferentes configuraciones de las pruebas necesarias para calcular este parámetro (Bachtiar et al. 2017). Esta diversidad representa una ventaja para fines de cálculo ingenieril y para el diseño de productos a base de madera; sin embargo, se requiere considerar los resultados especie por especie.

Para minimizar el efecto de la propagación del ultrasonido en un medio finito, la distancia entre los sensores, es decir, la distancia de recorrido (d, Fig. 1), debe ser mayor a la longitud de onda ( $\lambda$ ) (Bartholomeu, Gonçalves y Bucur, 2003). En esta investigación, las distancias entre sensores para las direcciones radial y tangencial (Fig. 1) son de 0,05 m, cantidad menor que la longitud de onda promedio en esta dirección (Tabla 4). En cambio, la distancia correspondiente a la dirección longitudinal de la madera sólida satisface este requerimiento, pero para el caso de la madera laminada la distancia de recorrido es $10 \%$ mayor que la magnitud de la longitud de onda. De tal forma, si se considera la irregularidad mencionada, la configuración de las pruebas de ultrasonido es adecuada para caracterizar la madera de $P$. pseudostrobus. 

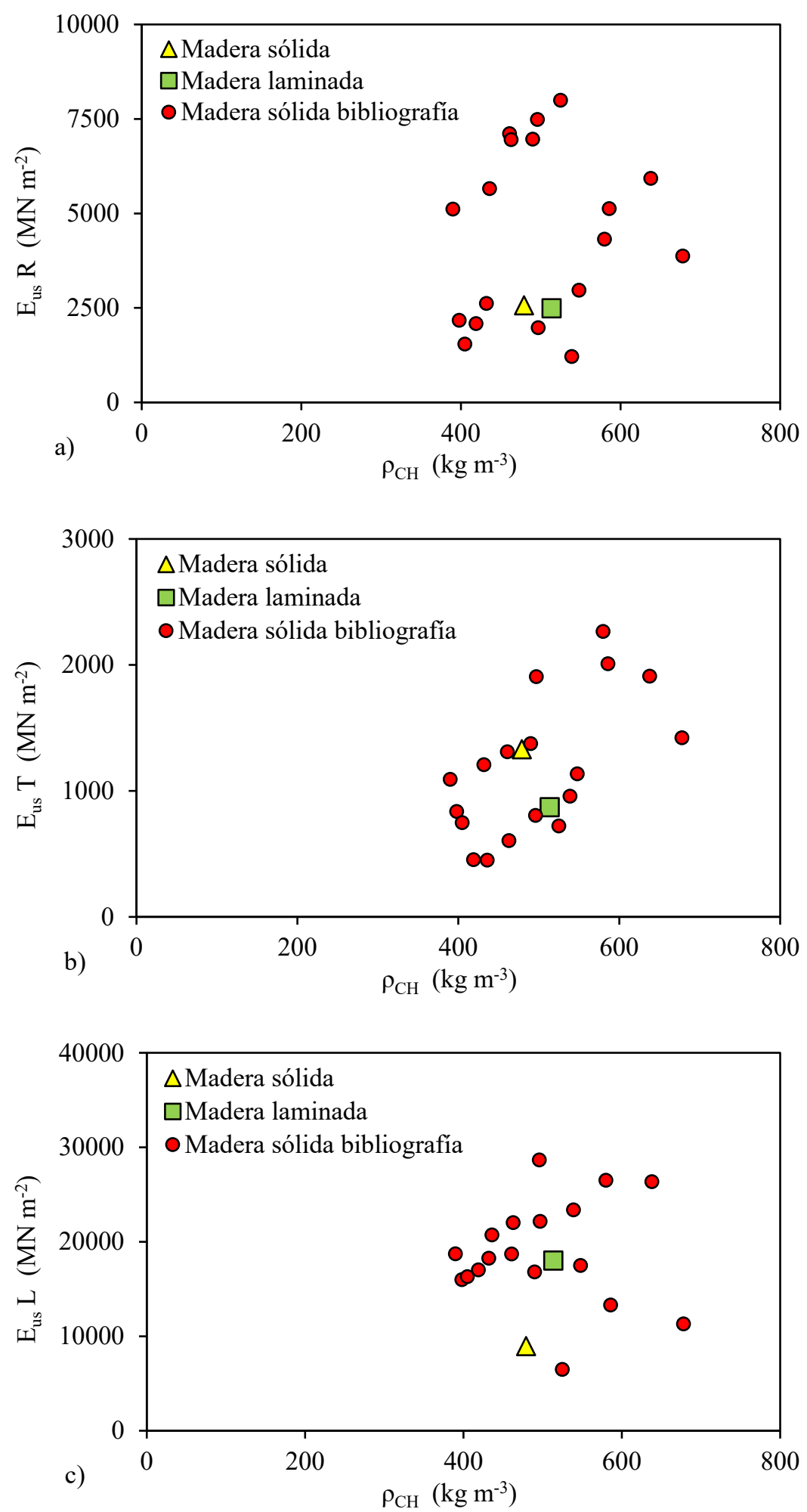

Figura 5. Dispersión del módulo dinámico de madera de Pinus pseudostrobus en función de su densidade. a) Dirección radial; b) Dirección tangencial; c) Dirección longitudinal. 
TABLA 4. Longitudes de onda y distancias de recorrido del ultrasonido en madera de Pinus pseudostrobus.

\begin{tabular}{cccccc}
\hline & Madera sólida & \multicolumn{4}{c}{ Madera laminada } \\
$\lambda_{R}$ & $\lambda_{T}$ & $\lambda_{\mathrm{L}}$ & $\lambda_{R}$ & $\lambda_{\mathrm{T}}$ & $\lambda_{\mathrm{L}}$ \\
$(\mathrm{m})$ & $(\mathrm{m})$ & $(\mathrm{m})$ & $(\mathrm{m})$ & $(\mathrm{m})$ & $(\mathrm{m})$ \\
0,043 & 0,031 & 0,079 & 0,041 & 0,024 & 0,110 \\
\hline & Madera sólida & & & Madera laminada & $\mathrm{d}_{\mathrm{L}}$ \\
$\mathrm{d}_{\mathrm{R}}$ & $\mathrm{d}_{\mathrm{T}}$ & $\mathrm{d}_{\mathrm{L}}$ & $\mathrm{d}_{\mathrm{R}}$ & $\mathrm{d}_{\mathrm{T}}$ & $(\mathrm{m})$ \\
$(\mathrm{m})$ & $(\mathrm{m})$ & $(\mathrm{m})$ & $(\mathrm{m})$ & $(\mathrm{m})$ & 0,05 \\
0,05 & 0,05 & 0,10 & 0,05 & 0,05 & 0,10 \\
\hline
\end{tabular}

$\lambda$ = longitud de onda; $d$ = distancia de recorrido del ultrasonido; $R=$ dirección radial; $T$ = dirección tangencial; $L=$ dirección longitudinal .

La influencia de la variabilidad de la madera a nivel microanatómico, que comprende células y fibras, puede moderarse eligiendo la frecuencia del emisor para que las longitudes de onda en el material se encuentren en un intervalo entre la dimensión máxima de los elementos anatómicos y la dimensión mínima de la muestra (Bucur y Declercq, 2006). En esta investigación, el diámetro máximo de las células/fibras en el plano radial-tangencial se estima en 0,001 m y el largo de las células/fibras en la dirección longitudinal en 0,005 m (Guitard y Gachet, 2004; Richter, Grosser, Heinz y Gasson, 2004; Wheeler, Baas y Gasson, 2007). Estos datos son menores que las dimensiones de las aristas de las probetas en las direcciones radial y tangencial que son de $0,05 \mathrm{~m}$ y de $0,10 \mathrm{~m}$ para la dirección longitudinal.

\section{CONCLUSIONES}

Se encontró experimentalmente que las densidades, las velocidades del ultrasonido y los módulos dinámicos de la madera laminada de $P$. psendostrobus son similares a las de madera sólida de esta especie. Igualmente, se corroboró que el tratamiento de laminado aumenta las velocidades y los módulos dinámicos en la dirección longitudinal, no así para las direcciones radial y tangencial. Además, la variabilidad en las características estudiadas disminuye como efecto de reconstituir madera sólida.

El aporte principal de este estudio radica en proporcionar datos de referencia e informar que la madera de esta especie, sólida y laminada, puede ser caracterizada con un método no destructivo. Para su posible aplicación en diseño estructural, es necesario efectuar pruebas estandarizadas en piezas con dimensiones de empleo.

\section{RECONOCIMIENTOS}

La investigación estuvo patrocinada por la Coordinación de la Investigación Científica de la Universidad Michoacana de San Nicolás de Hidalgo.

\section{REFERENCIAS}

Araujo Molina, O., Cerón Cardeña, M., Chan Martín, M., \& Azueta García, M. (2005). Resistencia a la flexión de vigas laminadas con tres especies de madera tropical mexicana. Ingeniería, 9(1), 5-12.

Baar, J., Tippner, J., \& Gryc, V. (2012). The influence of wood density on longitudinal wave velocity determined by the ultrasound method in comparison to the resonance longitudinal method. European Journal of Wood and Wood Products, 70(5), 767- 769. doi: 10.1007/s00107-011-0550-2

Bachtiar, E. V., Sanabria, S. J., Mittig, J. P., \& Nimes, P. (2017). Moisturedependent elastic characteristics of walnut and cherry wood by means of mechanical and ultrasonic test incorporating three different ultrasound data evaluation techniques. Wood Science and Technology, 51(1), 47-67. doi: 10.1007/s00226-016-0851-z

Bartholomeu, A., Gonçalves R., \& Bucur, V. (2003). Dispersion of ultrasonic waves in Eucalyptus lumber as a function of the geometry of boards. Scientia Forestalis, 63, 235-240.

Bourreau, D., Aimene, Y., Beauchêne, J. \& Thibaut, B. (2013). Feasibility of glued laminated timber beams with tropical hardwoods. European Journal of Wood and Wood Products, 71, 653-662. doi: 10.1007/s00107-013-0721-4 
Brémaud, I. (2012). Acoustical properties of wood in string instruments soundboards and tuned idiophones: Biological and cultural diversity. Journal of the Acoustical Society of America, 131(1), 807-818. doi: $10.1121 / 1.3651233$

Brémaud, I., Gril, J., \& Thibaut, B. (2011). Anisotropy of wood vibrational properties: dependence on grain angle and review of literature data. Wood Science and Technology, 45(4), 735-754. doi: $10.1007 / \mathrm{s} 00226-010-0393-8$

Bucur, V., \& Declercq, N. (2006). The anisotropy of biological composites studied with ultrasonic technique. Ultrasonics, 44, 829831. doi: $10.1016 /$ j.ultras.2006.05.203

Burdurlu, E., Kilic, M., Ilce, A. C., \& Ozan, U. (2007). The effects of ply organization and loading direction on bending strength and modulus of elasticity in laminated veneer lumber (LVL) obtained from beech (Fagus orientalis L.) and lombardy poplar (Populus nigra L.). Construction and Building Materials, 21(8), 1720-1725. doi: 10.1016/j.conbuildmat.2005.05.002

Dackermann, U., Elsener, R., Li, J., \& Crews, K. (2016). A comparative study of using static and ultrasonic material testing methods to determine the anisotropic material properties of wood. Construction and Building Materials, 102, 963-976. doi: 10.1016/j.conbuildmat.2015.07.195

Dietsch, P., \& Tannert, T. (2015) Assessing the integrity of gluedlaminated timber elements. Construction and Building Materials, 101(2), 1259-1270. doi: 10.1016/j.conbuildmat.2015.06.064

Ettelaei, A., Layeghi, M., Hosseinabadi, H. Z., \& Ebrahimi, G. (2019). Prediction of modulus of elasticity of poplar wood using ultrasonic technique by applying empirical correction factors. Measurement, $\quad$ 135, 392-399. doi: 10.1016/j.measurement.2018.11.076

Gaff, M. \& Gáborik, J. (2014). Effect of Cyclic Loading on the Elasticity of Beech Solid and Laminated Wood. BioResources, 9(3), 42884296. doi: 10.15376/biores.9.3.4288-4296

Guitard, D., \& Gachet, C. (2004). Paramètres structuraux et/ou ultrastructuraux facteurs de la variabilité intra-arbre de l'anisotropie élastique du bois. Annals of Forest Science, 61(2), 129139. doi: 10.1051 / forest:2004004

International Organization for Standardization [ISO] (2014a). ISO 13061-1:2014. Physical and mechanical properties of wood. Test methods for small clear wood specimens. Part 2: Determination of moisture content for physical and mechanical tests. Geneva: International Organization for Standardization. $6 \mathrm{p}$.

International Organization for Standardization [ISO] (2014b). ISO 13061-2:2014. Physical and mechanical properties of wood. Test methods for small clear wood specimens. Part 2: Determination of density for physical and mechanical tests. Geneva: International Organization for Standardization. 5 p.

Ho, S. C. (2017). Anisotropy of softwood structural lumber using the elastic modulus determined by the ultrasonic nondestructive method. Journal of the Korean Wood Science and Technology, 45(1), 20 27. doi: 10.5658/WOOD.2017.45.1.20

Kandler, G., Lukacevic, M., Zechmeister, C., Wolff, S., \& Füssl, J. (2018). Stochastic engineering framework for timber structural elements and its application to glued laminated timber beams. Construction and Building Materials, 190, 573-592. doi: 10.1016/j.conbuildmat.2018.09.129

Kwon, J. H., Shin, R. H., Ayrilmis, N., \& Han, T. H. (2014). Properties of solid wood and laminated wood lumber manufactured by cold pressing and heat treatment. Materials and Design, 62, 375-381. doi: 10.1016/j.matdes.2014.05.032

Liew, K. C. \& Singan, G. (2016). Engineered wood composite of laminated veneer lumber: Physical and mechanical properties. Materials Science Forum, 842, 103-128. doi: 10.4028/www.scientific.net/MSF.842.103

Mvondo, R. R. N., Meukam, P., Jeong, J., Meneses, D. D., \& Nkeng, E.G. (2017). Influence of water content on the mechanical and chemical properties of tropical wood species. Results in Physics, 7 , 2096-2103. doi: 10.1007/s00231-019-02795-8

Nadir, Y., \& Nagarajan, P. (2014). The behavior of horizontally glued laminated beams using rubber Wood. Construction and Building Materials, 55, 398-405. doi: 10.1016/j.conbuildmat.2014.01.032

Perré, P., Almeida, G., Ayouz, M., \& Frank, X. (2016). New modelling approaches to predict wood properties from its cellular structure: image-based representation and meshless methods. Annals of Forest Science, 73(1), 147-162. doi: 10.1007/s13595-015-0519-0

Ribeiro, A. S., De Jesus, A. M. P., Lima, A. M., \&. Lousada, J. L. C. (2009). Study of strengthening solutions for glued-laminated wood beams of maritime pine wood. Construction and Building Materials, 23, 2738-2745. doi: 10.1016/j.conbuildmat.2009.02.042

Richter, H. G., Grosser, D., Heinz I., \& Gasson, P. E. (2004). IAWA list of microscopic features for softwood identification. IAWA Journal, 25(1), 1-70. doi: 10.1163/22941932-90000349

Schubert, S., Gsell, D., Dual, J., Motavalli, M., \& Niemz, P. (2009). Acoustic wood tomography on trees and the challenge of wood heterogeneity. Holzforschung, 63(1), 107-112. doi: 10.1515/HF.2009.028

Sotomayor Castellanos, J. R. (2018). Densidad y módulos dinámicos por vibraciones transversales de madera sólida y laminada de tres especies tropicales. Tecnociencia, 20(2), 71-90. Recuperado de https://www.researchgate.net/publication/331101383_Densida 
d_y_modulos_dinamicos_por_vibraciones_transversales_de_ma dera_solida_y_laminada_de_tres_especies_tropicales

Sotomayor Castellanos, J. R. (2019). Módulo dinámico longitudinal determinado por ultrasonido en madera de tres angiospermas sólida y laminada. Entreciencias, 7(21), 23-35. doi: 10.22201/enesl.20078064e.2019.21.68151

Sotomayor Castellanos, J. R. (2020). Caracterización mecánica por ondas de esfuerzo de la madera laminada de tres especies angiospermas. Brazilian Journal of Wood Science, 11(1), 32-41. doi: 10.12953/21776830/rcm.v11n1p32-41

Sotomayor Castellanos, J. R., \& Ruiz Aquino, F. (2017). Caracterización dinámica de madera laminada. Investigación e Ingeniería de la Madera, 13(3), 22-44.

Sotomayor Castellanos, J. R., Adachi, K., Iida, R., \& Hayashi, T. (2020). Módulos de elasticidad y de ruptura de madera laminada de tres especies tropicales. Acta Universitaria, 29, 1-15. doi: $10.15174 /$ au. 2019.2178

Sotomayor Castellanos, J. R., Carmona Delgado, I., Cervantes Móreles, I., Garduño Suárez, D., Jiménez Guzmán, D. Z., Lemus Durán, R., Maldonado Correa, D., Pérez Tello, A., Vaca Hernández, M. A., \& Valdez Velázquez, O. (2015). Madera laminada de Pinus pseudostrobus. Caracterización dinámica con métodos no destructivos. Investigación e Ingeniería de la Madera, 11(3), 4-34.

Sotomayor Castellanos, J. R., García Mariscal, J. L., Moya Lara, C. E., \& Olguín Cerón, J. B. (2010a). Higroscopía y anisotropía de la madera de Pinus michoacana, Pinus douglasiana y Pinus pringlei. Higrocontracción, velocidad del ultrasonido y módulo de elasticidad dinámico. Investigación e Ingeniería de la Madera, 6(3), 332.
Sotomayor Castellanos, J. R., Guridi Gómez, L. I., \& García Moreno, T. (2010b). Características acústicas de la madera de 152 especies mexicanas. Velocidad del ultrasonido, módulo de elasticidad, índice material y factor de calidad. Base de datos. Investigación e Ingeniería de la Madera. 6(1), 3-32.

Wheeler, E. A., Baas P., \& Gasson, P. E. (2007). IAWA list of microscopic features for hardwoods identification. IAWA Bulletin, 10(3), 219-332. doi: 10.1163/22941932-90000496

Zhang, T., Bai, S. L., Bardet, S., Alméras, T., Thibaut, B., \& Beauchêne, J. (2011). Radial variations of vibrational properties of three tropical woods. Journal of Wood Science, 57(5), 377-386. doi: 10.1007/s10086-011-1189-7

Manuscrito recibido el 21 de agosto de 2020

Aceptado el 17 de abril de 2021

Publicado el 30 de diciembre de 2021

Este documento se debe citar como:

Macedo Alquicira, I., \& Sotomayor Castellanos, J. R. (2021). Densidad, velocidad del ultrasonido y módulo dinámico de madera sólida y laminada de Pinus pseudostrobus. Madera y Bosques, 27(3), e2732235. doi: 10.21829/myb.2021.2732235

Madera y Bosques por Instituto de Ecología, A.C. se distribuye bajo una Licencia Creative Commons Atribución-NoComercialCompartirlgual 4.0 Internacional. 